\title{
Long term outcome of vertical expandable prosthetic titanium rib treatment in children with early onset scoliosis
}

\author{
Daniel Studer, Carol-Claudius Hasler \\ Department of Orthopaedic, Children's Hospital Basel, University of Basel, Basel, Switzerland \\ Contributions: (I) Conception and design: All authors; (II) Administrative support: All authors; (III) Provision of study materials or patients: All \\ authors; (IV) Collection and assembly of data: All authors; (V) Data analysis and interpretation: All authors; (VI) Manuscript writing: All authors; (VII) \\ Final approval of manuscript: All authors. \\ Correspondence to: Daniel Studer, MD. Department of Orthopaedic, Children's Hospital Basel, University of Basel, Spitalstrasse 33, 4056 Basel, \\ Switzerland. Email: danielstuder19@gmail.com.
}

\begin{abstract}
The vertical expandable prosthetic titanium rib (VEPTR) device was originally developed for the treatment of thoracic insufficiency syndrome with the aim of improving respiratory function of affected patients. Although clinically obvious, the changes in pulmonary function of VEPTR-treated patients are difficult to assess when using common lung function tests, and newer techniques based on functional magnetic resonance imaging (MRI) are currently being evaluated. The potential of improving lung function and simultaneously controlling the spinal deformity has continuously broadened the spectrum of indications for VEPTR, not least due to the frequent reports of complications with spine-based traditional growing rods (tGR). However, the initial enthusiasm of spine-sparing deformity correction has progressively subsided with the increasing number of reports on complications, including the detection of extraspinal ossifications along the implants and across ribs. The avoidance of repetitive surgical implant lengthening with the availability of motorized distraction-based implants has further diminished the use of VEPTR, especially in the absence of volume-depletion deformities of the thorax. In view of the still scarce reporting on the ultimate strategy of VEPTR treatment and the lack of long-term follow-up of patients receiving growth-sparing surgery, only limited conclusions can be drawn so far. Based on the available reports, however, the intended deformity corrections with final fusion surgeries can be achieved to a rather limited extent, while the complication and reoperation rates are still very high.
\end{abstract}

Keywords: Early onset scoliosis (EOS); growth-sparing treatment; thoracic insufficiency syndrome; graduates

Submitted Aug 23, 2019. Accepted for publication Sep 27, 2019.

doi: 10.21037/atm.2019.09.158

View this article at: http://dx.doi.org/10.21037/atm.2019.09.158

\section{Introduction}

To understand the vertical expandable prosthetic titanium rib (VEPTR) concept, the philosophy behind the device must first be known and understood. According to the inventor, Dr. Robert Campbell Jr., the VEPTR story started with the treatment of an 8-month-old ventilator-dependent child with rib agenesis and scoliosis who's respiratory function could significantly be improved by means of a Steinmann pin chest wall prosthesis (1). The expansion of the thorax with the pins permitted weaning from the respirator but led to problems with the further growth of the child due to the tethering effect of the non-expandable implant. The fact that the Steinmann pins had to be regularly replaced surgically by complex and complicating operations led to the idea of an expandable implant and thus "The Titanium Rib Project" was born.

\section{Thoracic insufficiency syndrome (TIS)}

Despite the different underlying diseases of the subsequently treated children, it became clear that there were common issues: the considerable deformities negatively impaired 

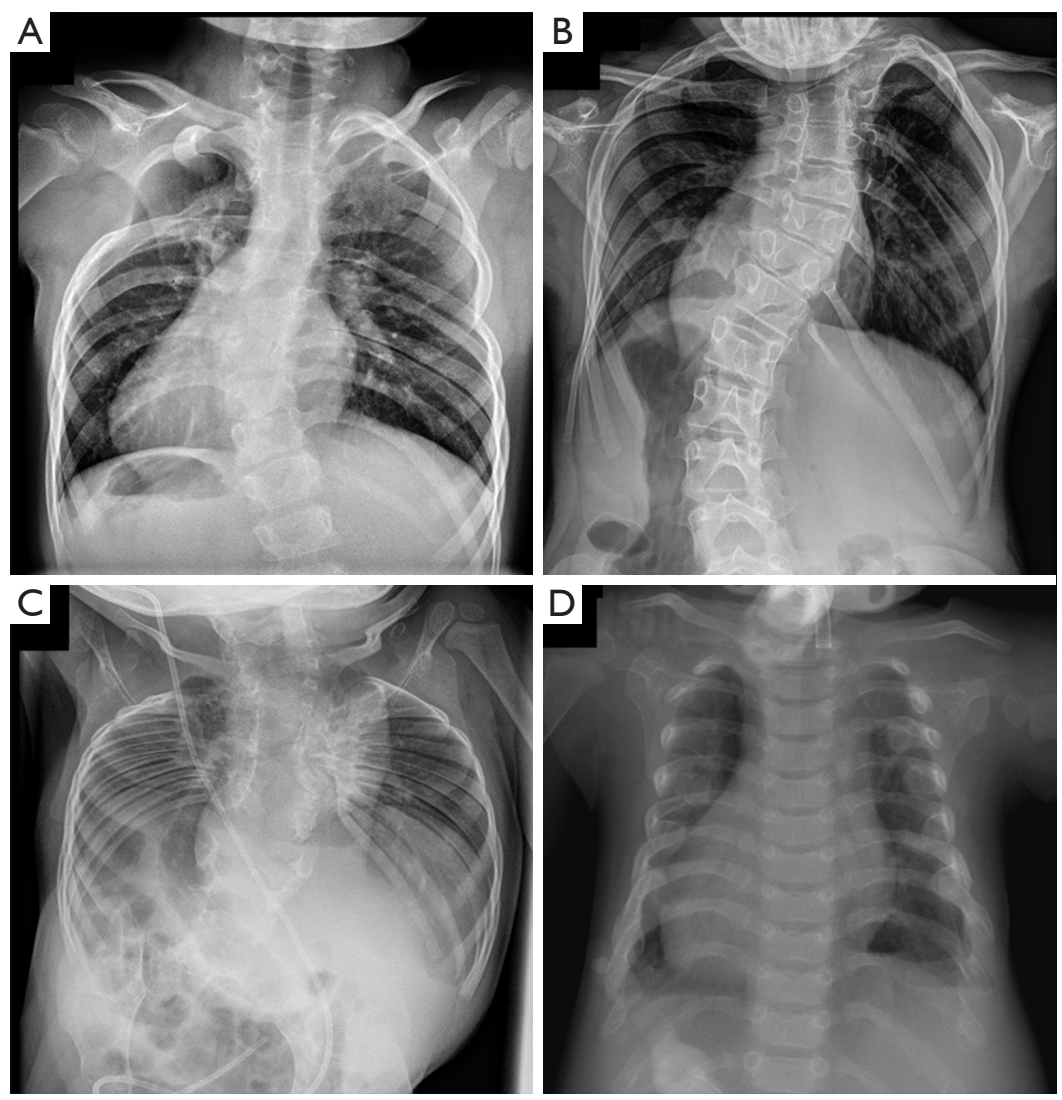

Figure 1 The four different types of volume-depletion deformities (VDD). (A) Type I VDD with absent ribs and scoliosis; (B) type II VDD with fused ribs and scoliosis; (C) type IIIa VDD in a patient with Jarcho-Levin syndrome with a foreshortened thorax; (D) type IIIb VDD in a patient with Jeune syndrome and a transverse constricted thorax.

lung function by limiting the space available for the lungs by reducing the growth of the rib cage and the thoracic spine (the thorax), as well as restricting the rib cage's ability to expand the lungs with respiration. These findings led to the introduction of the term "thoracic insufficiency syndrome" (TIS), which describes the inability of the thorax to support normal respiration or lung growth (2). Later, the term was further divided into a primary (abnormalities of the thorax, including congenital structural problems of the thoracic spine, rib cage, and the diaphragm) and a secondary TIS (relative obstruction to the excursion of the diaphragm due to either lumbar kyphosis or pelvic obliquity) (3).

\section{Indication for VEPTR}

The primary indication for VEPTR is the presence of TIS in skeletally immature patients. From an anatomical point of view, in addition to absent ribs, constrictive chest wall syndrome (including fused ribs and scoliosis), hypoplastic thorax, or early onset scoliosis (EOS) of congenital or neurogenic origin without rib anomalies also qualify for VEPTR treatment. The adequate VEPTR expansion thoracoplasty strategy is based on the three-dimensional type of volume-depletion deformity (VDD) of the thorax (4) (Figure 1).

Type I is characterised by absent ribs and scoliosis, resulting in a unilateral thoracic hypoplasia. The lung prolapses into the chest with progressing loss of lung volume. In type II VDD, scoliosis is associated with fused ribs leading to a constriction and shortening of the affected hemithorax. A foreshortened thorax with bilateral longitudinal constriction of the lungs, as in Jarcho-Levin syndrome, is categorized as type IIIa, and a transverse constricted thorax, as in Jeune asphyxiating thoracic dystrophy, is described as type IIIb. For type I VDD, VEPTR expansion thoracoplasty is considered a stabilization expansion thoracoplasty. The operative goal is to increase the space available for the lung by stabilizing the flail chest wall segment and laterally expanding and 



Figure 2 Recommended VEPTR treatment techniques dependent on the type of volume-depletion-deformity (VDD). VEPTR, vertical expandable prosthetic titanium rib.

lengthening the collapsed hemithorax, usually using two ribto-rib VEPTR devices. An opening wedge thoracostomy is performed for type II VDD using a rib-to-rib VEPTR to stabilize the correction. An additional hybrid VEPTR (ribto-lumbar spine or pelvis) is used depending on age of the patient, severity of the deformity, and the development of an adjacent compensatory thoracolumbar or lumbar curve. Staged bilateral opening wedge thoracostomies for type IIIa VDD and staged bilateral dynamic segmental expansion thoracoplasties for type IIIb VDD are recommended (Figure 2).

Despite these concrete technical guidelines for the use of VEPTR, there is a great variability in the choice of implants and construct type, even among experts (5). Moreover, for patients with severe EOS, a bilateral use of VEPTR attached to the pelvis has been described as a viable treatment option, potentially limiting spontaneous fusion of the spine (6).

\section{Results and outcome during growth-sparing VEPTR treatment}

Early reports on outcomes with VEPTR treatment confirm increased volume of the constricted hemithorax (CT-derived lung volumes), good control of the spinal deformity, and normal growth of the thoracic spine (7). In addition, relevant functional and cosmetic improvements have also been documented (8). Despite a clinically and radiographically apparent geometrical expansion of the thorax after VEPTR insertion, no similar improvement in lung function could be confirmed by Mayer and Redding in their investigation on early changes in pulmonary function after VEPTR insertion (9). They concluded that the lack of change in pulmonary function suggested that the benefit of VEPTR insertion may lie more in stabilizing the thorax and improving respiratory mechanics, which are not assessed by common lung function tests. In 2013, Campbell, in his review on past experience and the future of VEPTR principles, explained that TIS, which could not be captured with current imaging modalities, can possibly be defined more objectively using dynamic lung MRI in the future (1). A recent article on quantitative dynamic MRI of pediatric patients with TIS concluded that a significant increase in post-operative tidal volumes suggests a treatmentrelated improvement in lung capacity, and that a reduction of resting breathing rate following surgery indicates that breathing function was improved (10). Quantitative dynamic MRI parameters may therefore offer an objective marker set for studying TIS warranting further research in this field.

Over time, the range of indications for the use of VEPTR has been extended, not least because of the high complication rates and the detection of spontaneous autofusion of the vertebral column in spine-based growthguiding techniques (11-13). Starting in 2002, the University Children's Hospital Basel (UKBB) was the first institution outside the USA to use VEPTR. An analysis on 23 of our own patients in 2010 demonstrated VEPTR to be a valuable alternative to dual growing rods for non-congenital EOS even without rib fusions (14). Although the correction of 

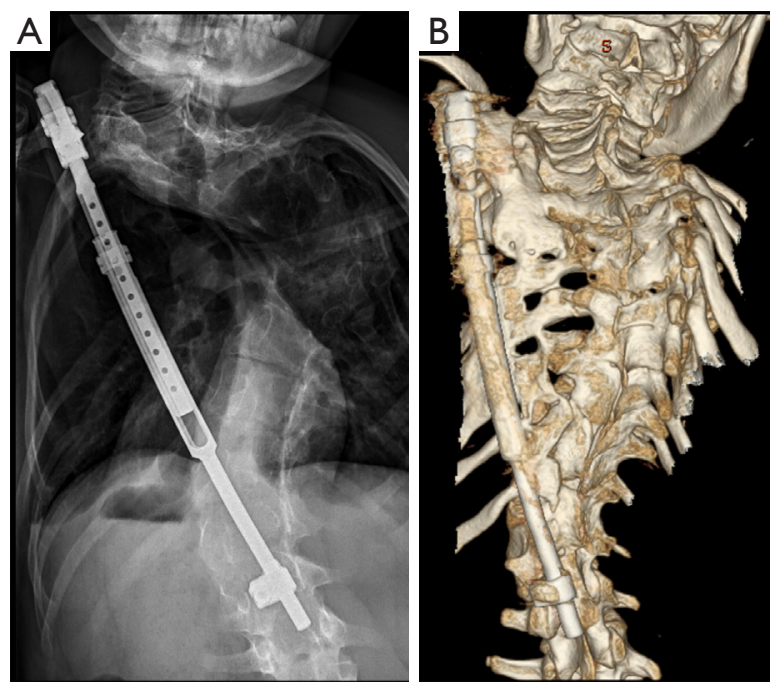

Figure 3 Plain X-ray (A) and CT (B) of a 15-year-old boy treated with VEPTR for congenital early onset scoliosis. Although hardly visible on plain $\mathrm{X}$-ray, there are obvious ossifications visible on CT scan around the proximal anchors, along the implant, and even across the ribs. VEPTR, vertical expandable prosthetic titanium rib.

the coronal plane deformity was lower, the control of the sagittal plane deformity and of the pelvic obliquity was comparable, and the complication rate was lower compared to traditional growing rods (tGR). Equal conclusions were drawn by El-Hawary et al. in their multicentre study on VEPTR treatment in children with EOS without rib abnormalities (15). They found that 54/63 patients (86\%) had a successful outcome, which was defined as a curve magnitude less than or equal to the preoperative magnitude at 2-year follow-up. Apart from satisfactory deformity control, they also reported on continuous spine growth with VEPTR treatment in their heterogeneous population without rib abnormalities.

With longer follow-up times, publications reporting of increasing complication rates with the use of VEPTR have become more frequent. In a retrospective comparison of complications among growth sparing implants by Sankar et al., both spine-based dual growing rods and VEPTR had an average of 0.52 year complications with an increasing complication rate with passage of time for both systems (16). El-Hawary et al., in their previously mentioned multicentre study, reported that $49 \%$ of patients had a least one complication, the majority being implant-related (26\%), with the rest being related to wound complications and pneumonia (15). Campbell himself together with Smith reported a $3.3 \%$ infection rate per VEPTR surgery (4). The need for repetitive surgical device lengthening is considered the main risk factor for implant-related infections. In a prospective study, we investigated the amount of bacterial implant colonization in 39 VEPTR patients (17). Removed implant components during 163 lengthening procedures were treated by sonication, and the sonication fluid was subsequently inoculated onto aerobic and anaerobic agar plates. Bacterial colonization of the implants was observed in $46 \%$ of patients, and 4 patients $(10 \%)$ developed an implant-related surgical site infection during this observation period.

Apart from complication rates being comparable to other distraction-based systems, additional problems that were hoped to be avoided with spine-sparing implants have nevertheless occurred. In a retrospective international multicentre study, a radiographic analysis focusing on extraspinal ossifications of 65 VEPTR patients with a minimum follow-up of 4 years was performed (18). Ossifications were classified as type I (at the anchor points), type II (along the central part of the VEPTR), or type III (re-ossification after thoracostomy). It was reported that $65 \%$ of patients showed ossifications, most of them being type I and/or type II (Figure 3). The possible negative impact on chest wall compliance and spinal mobility, especially in type II ossifications, was mentioned but not further investigated.

The growth-stimulating effect, commonly documented by a simplified 2-dimensional assessment of T1-S1 or T1T12 distance on AP radiographs, has repeatedly been confirmed. These measurements, however, are limited by the projectional nature of a spine radiograph and the ignorance of more detailed regional growth phenomenon. We therefore performed a retrospective radiographic study with a minimum 4-year follow-up to further investigate the effect of year-long distraction-based EOS treatment by VEPTR on vertebral growth modulation (19). Two groups of nine patients each with EOS, all displaying normally segmented and shaped lumbar vertebrae at the beginning of VEPTR treatment were compared. Group 1 consisted of patients with VEPTR constructs spanning the lumbar spine, whereas patients in group 2 all had VEPTR constructs leaving the lumbar spine uninstrumented. Although the gain in vertebral body height $(\mathrm{VBH})$ of the lumbar segments did not differ significantly between groups, patients in group 1 had significantly less growth in regards to vertebral depth (VD) (Figure 4).

This altered vertebral height-to-depth ratio (H/D ratio) led to an impressive change in vertebral morphology, likely 

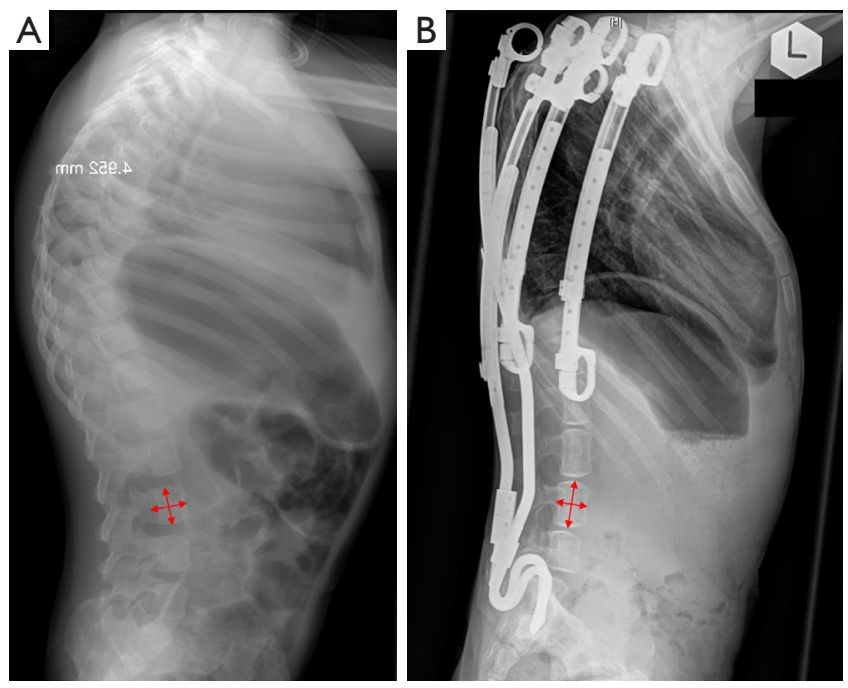

Figure 4 Altered vertebral morphology. (A) A lateral whole spine $\mathrm{X}$-ray of 1.5-year-old female with syndrome-related early onset scoliosis (EOS); (B) the same patient after 6 years of growthsparing treatment with bilateral VEPTR constructs, spanning the lumbar spine. The red arrows point to the clear morphological changes of the lumbar vertebrae with a significant increase in the vertebral height-to-depth ratio caused by the year-long VEPTR treatment. VEPTR, vertical expandable prosthetic titanium rib.

as a result of year-long stress-shielding of the spanned lumbar segments. The biomechanical consequences of this metamorphosis into high and slender vertebral bodies in combination with worrisome disc changes remain unclear and have not yet been studied in detail.

\section{Results and outcome at the end of VEPTR treatment}

Despite the progress made, growth-sparing surgery for EOS patients with distraction-based implants predominantly remains a delaying-fusion strategy. While there are numerous reports on results and outcome during ongoing VEPTR treatment, not much information on definitive procedures at the end of growth in EOS patients is available. The paucity of reliable data and the heterogeneity of EOS patients restricts the decision-making process for so-called "graduates" of growth-sparing surgery. Many times, the decision about when to stop expansions is less surgeondriven, but rather determined by curve progression, failure to further distract, or complications (20,21). In 2013, Flynn et al. were the first to report a multicentre series of 99 graduates of tGR treatment (22). Two other reports from

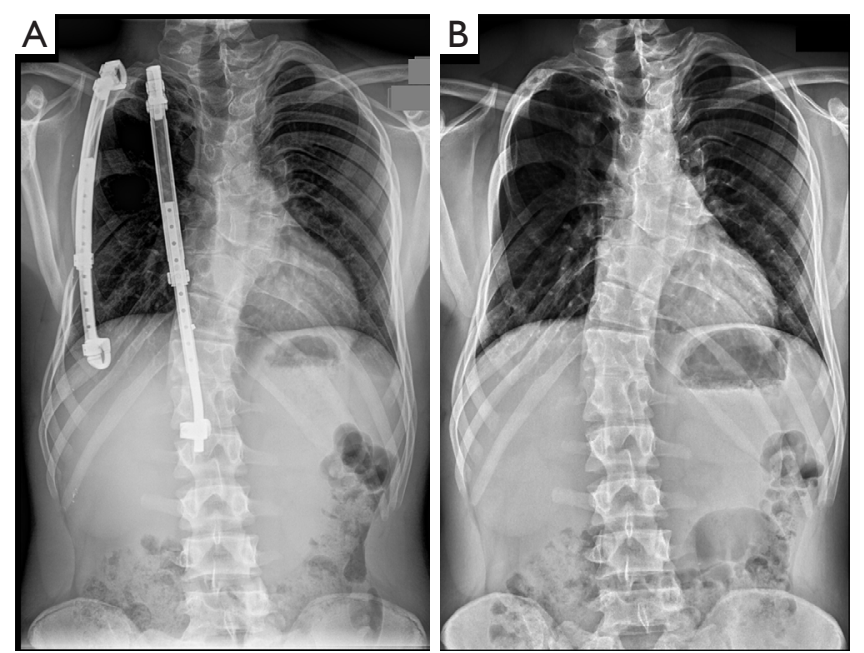

Figure 5 Anteroposterior spine X-rays of a patient with type II volume-depletion deformity (VDD) at the end of growth-sparing treatment with VEPTR before (A) and 23 months after (B) implant removal. VEPTR, vertical expandable prosthetic titanium rib.

the Growing Spine Study Group (GSSG) focused on EOS patients after tGR and Shilla growth guidance treatment, with the former focusing on the possibility of avoiding final surgical fusion $(23,24)$. Johnston et al. reported on the functional and radiographic outcomes of 12 patients following growth-sparing management, including one VEPTR patient, while Sawyer et al. looked at complications and radiographic outcomes of 37 patients after distractionbased treatment, in which 32 had rib-based fixation without specification of the type of implant $(25,26)$. Regardless of the type of implant, the results were mostly modest with frequently low correction potential and high complication and reoperation rates with final fusion. Recently, we published a study on the radiographic outcome and complication rate of 34 of our own VEPTR graduates (27). The mean age at the end of VEPTR treatment was 13.8 years, with $50 \%$ of the graduates following a non-fusion strategy. In 5 patients with congenital EOS and unilateral VEPTR constructs the implants were simply removed (Figure 5), and in 12 patients the VEPTR was left in situ. So far, no further complications have been observed over an average follow-up period of 41 months in the non-fusion group.

Seventeen patients underwent final fusion surgery (Figure 6).

Younger age at first VEPTR implantation and higher major curve's and main kyphosis' Cobb angles at the end of 

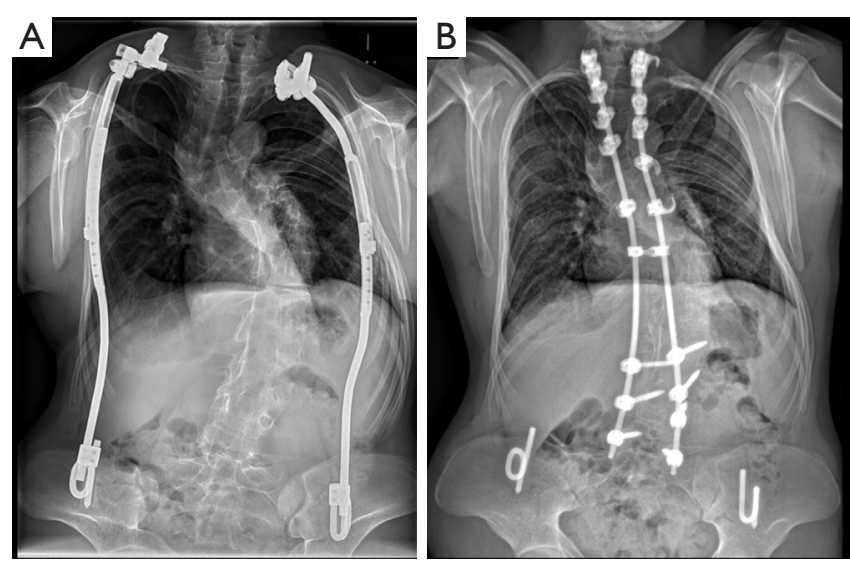

Figure 6 Anteroposterior spine X-rays of a patient with congenital early onset scoliosis at the end of growth-sparing treatment with VEPTR (A) and 27 months after undergoing final fusion surgery (B). The stiffened spine and thorax after year-long distractionbased treatment allowed for only very limited deformity correction with final fusion surgery. VEPTR, vertical expandable prosthetic titanium rib.

VEPTR treatment were parameters that reached statistical significance between patients undergoing final fusion surgery and patients following a non-fusion strategy. There was a $41 \%$ complication rate in the final fusion group over a mean follow-up period of 25 months, leading to 6 unplanned returns to the operating room. For final fusion surgery, a $14 \%$ average correction rate of the main coronal deformity was achieved. This is even lower compared to the limited correction rate reported by Flynn et al. in their series of tGR graduates (22) and might be explained by the presence of ossifications of the thorax in rib-based implants in addition to the spontaneous autofusions of the spine. The considerable complication rate and occurrence of rigid deformities of the spine and the thorax suggest that final fusion procedures should be avoided. Satisfactory axial alignment and balance are minimum prerequisites for this, but the current lack of mid- and long-term follow-up of graduates without final fusion do not yet allow any firm conclusion to be drawn (24).

\section{The future of VEPTR}

With the availability of motorized growth-sparing implants which reduce the heavy psycho-social burden of repetitive surgical implant lengthening, and considering the results described above, the use of VEPTR implants has consistently been narrowed to the original indication for the treatment of TIS. This, to us, appears correct and should be the role of VEPTR in the future. Reports on long-term follow-up of patients after completed VEPTR treatment are still pending, and the most important prerequisite for the development of an evidence-based algorithm in the decision-making process for the final strategy at the end of growth has also not been established.

\section{Acknowledgments}

None.

\section{Footnote}

Conflicts of Interest: The authors have no conflicts of interest to declare.

Ethical Statement: The authors are accountable for all aspects of the work in ensuring that questions related to the accuracy or integrity of any part of the work are appropriately investigated and resolved.

\section{References}

1. Campbell RM Jr. VEPTR: past experience and the future of VEPTR principles. Eur Spine J 2013;22 Suppl 2:S106-17.

2. Campbell RM, Smith MD, Mayes T, et al. The treatment of thoracic insufficiency syndrome scientific exhibit.

In: American academy of orthopaedic surgeons annual meeting, 1992.

3. Campbell RM Jr, Smith MD, Mayes TC, et al. The effect of opening wedge thoracostomy on thoracic insufficiency syndrome associated with fused ribs and congenital scoliosis. J Bone Joint Surg Am 2004;86:1659-74.

4. Campbell RM Jr, Smith MD. Thoracic insufficiency syndrome and exotic scoliosis. J Bone Joint Surg Am 2007;89 Suppl 1:108-22.

5. Vitale MG, Gomez JA, Matsumoto H, et al. Variability of expert opinion in treatment of early-onset scoliosis. Clin Orthop Relat Res 2011;469:1317-22.

6. Samdani AF, Ranade A, Dolch HJ, et al. Bilateral use of the vertical expandable prosthetic titanium rib attached to the pelvis: a novel treatment for scoliosis in the growing spine. J Neurosurg Spine 2009;10:287-92.

7. Emans JB, Caubet JF, Ordonez CL, et al. The treatment of spine and chest wall deformities with fused ribs 
by expansion thoracostomy and insertion of vertical expandable prosthetic titanium rib: growth of thoracic spine and improvement of lung volumes. Spine (Phila $\mathrm{Pa}$ 1976) 2005;30:S58-68.

8. Hell AK, Campbell RM, Hefti F. The vertical expandable prosthetic titanium rib implant for the treatment of thoracic insufficiency syndrome associated with congenital and neuromuscular scoliosis in young children. J Pediatr Orthop B 2005;14:287-93.

9. Mayer OH, Redding G. Early changes in pulmonary function after vertical expandable prosthetic titanium rib insertion in children with thoracic insufficiency syndrome. J Pediatr Orthop 2009;29:35-8.

10. Tong Y, Udupa JK, Wileyto EP, et al. Quantitative dynamic MRI (QdMRI) Volumetric Analysis of Pediatric Patients with Thoracic Insufficiency Syndrome. Proc SPIE Int Soc Opt Eng 2018. doi: 10.1117/12.2294048.

11. Bess S, Akbarnia BA, Thompson GH, et al. Complications of growing-rod treatment for early-onset scoliosis: analysis of one hundred and forty patients. J Bone Joint Surg Am 2010;92:2533-43.

12. Cahill PJ, Marvil S, Cuddihy L, et al. Autofusion in the immature spine treated with growing rods. Spine (Phila $\mathrm{Pa}$ 1976) 2010;35:E1199-203.

13. Sankar WN, Skaggs DL, Yazici M, et al. Lengthening of dual growing rods and the law of diminishing returns. Spine (Phila Pa 1976) 2011;36:806-9.

14. Hasler CC, Mehrkens A, Hefti F. Efficacy and safety of VEPTR instrumentation for progressive spine deformities in young children without rib fusions. Eur Spine J 2010;19:400-8.

15. El-Hawary R, Kadhim M, Vitale M, et al. VEPTR Implantation to Treat Children With Early-Onset Scoliosis Without Rib Abnormalities: Early Results From a Prospective Multicenter Study. J Pediatr Orthop 2017;37:e599-605.

16. Sankar WN, Acevedo DC, Skaggs DL. Comparison of complications among growing spinal implants. Spine (Phila Pa 1976) 2010;35:2091-6.

17. Plaass C, Hasler CC, Heininger U, et al. Bacterial colonization of VEPTR implants under repeated expansions in children with severe early onset spinal

Cite this article as: Studer D, Hasler CC. Long term outcome of vertical expandable prosthetic titanium rib treatment in children with early onset scoliosis. Ann Transl Med 2020;8(2):25. doi: 10.21037/atm.2019.09.158 deformities. Eur Spine J 2016;25:549-56.

18. Zivkovic V, Buchler P, Ovadia D, et al. Extraspinal ossifications after implantation of vertical expandable prosthetic titanium ribs (VEPTRs). J Child Orthop 2014;8:237-44.

19. Hasler CC, Studer D, Buchler P. Metamorphosis of human lumbar vertebrae induced by VEPTR growth modulation and stress shielding. J Child Orthop 2015;9:287-93.

20. Pizones J, Martin-Buitrago MP, Sanchez Marquez JM, et al. Decision Making of Graduation in Patients With EarlyOnset Scoliosis at the End of Distraction-Based Programs: Risks and Benefits of Definitive Fusion. Spine Deform 2018;6:308-13.

21. Yang JS, McElroy MJ, Akbarnia BA, et al. Growing rods for spinal deformity: characterizing consensus and variation in current use. J Pediatr Orthop 2010;30:264-70.

22. Flynn JM, Tomlinson LA, Pawelek J, et al. Growingrod graduates: lessons learned from ninety-nine patients who completed lengthening. J Bone Joint Surg Am 2013;95:1745-50.

23. Luhmann SJ, Smith JC, McClung A, et al. Radiographic Outcomes of Shilla Growth Guidance System and Traditional Growing Rods Through Definitive Treatment. Spine Deform 2017;5:277-82.

24. Jain A, Sponseller PD, Flynn JM, et al. Avoidance of "Final" Surgical Fusion After Growing-Rod Treatment for Early-Onset Scoliosis. J Bone Joint Surg Am 2016;98:1073-8.

25. Johnston CE, Tran DP, McClung A. Functional and Radiographic Outcomes Following Growth-Sparing Management of Early-Onset Scoliosis. J Bone Joint Surg Am 2017;99:1036-42.

26. Sawyer JR, de Mendonca RG, Flynn TS, et al. Complications and Radiographic Outcomes of Posterior Spinal Fusion and Observation in Patients Who Have Undergone Distraction-Based Treatment for Early Onset Scoliosis. Spine Deform 2016;4:407-12.

27. Studer D, Buchler P, Hasler CC. Radiographic Outcome and Complication Rate of 34 Graduates After Treatment With Vertical Expandable Prosthetic Titanium Rib (VEPTR): A Single Centre Report. J Pediatr Orthop 2019. [Epub ahead of print]. 